\title{
Indicadores de desempeño clave para unidades académicas universitarias*
}

\author{
Yáber, Guillermo** \\ Valarino, Elizabeth ${ }^{\star \star *}$
}

\section{Resumen}

El propósito de este trabajo es aplicar el modelo de Gerencia de Sistemas Conductuales (G.S.C.) y el enfoque sistémico de organización y gestión en una unidad académica universitaria. Se emplearon en el diseño, desarrollo, aplicación y evaluación de indicadores de desempeño clave en una unidad académica universitaria venezolana. Se utilizaron indicadores para las siguientes categorías de gestión: efecto, impacto, eficacia, eficiencia, calidad y economía. Los resultados obtenidos sugieren que la unidad académica mejora sus indicadores de efecto, impacto, y eficiencia a través del tiempo y tiene oportunidad de mejoras en las categorías de calidad y economía. Los datos para analizar la eficacia no estuvieron disponibles para el momento del estudio. Los resultados proporcionan pautas tanto para la formulación de políticas académicas como para la toma de decisiones y ejecución de acciones destinadas a corregir y mejorar el desempeño de esa dependencia universitaria. Estos indicadores y los enfoques de donde se derivan, pueden aplicarse a otras unidades académicas en la misma universidad o a otras instituciones de educación superior que ofrezcan títulos o grados académicos. Se concluye que medir y evaluar indicadores de gestión constituyen prácticas para instrumentar la autorregulación y el control de la gestión en las instituciones de educación superior.

Palabras clave: Indicadores, desempeño, unidades académicas, universidades.

Recibido: 02-04-24 . Aceptado: 02-09-30

Agradecemos a la Dra. Corina Groeger por sus valiosos comentarios a este trabajo.

** Ph.D. Profesor titular del Departamento de Ciencia y Tecnología del Comportamiento de la Universidad Simón Bolívar. E-mail: gyaber@usb.ve

*** Dra. Profesora titular del Departamento de Ciencia y Tecnología del Comportamiento de la Universidad Simón Bolívar. E-mail: evalarin@usb.ve 


\section{Key Indicators of Performance in Academic Units in Universities}

\section{Abstract}

The purpose of this paper is to apply the Conductive Management System (G.S.C.) model and the Systematic Organization and Management Focus to a university level academic unit. The design, development, application and evaluation of efficiency indicators were employed in an Venezuelan university academic unit. Indicators were employed in the following management categories: effect, impact, efficacy, efficiency, quality and economy. The results obtained indicated that the academic unit improved its levels of effect, impact and efficiency over time and could also improve the categories of quality and economy. Data as to efficacy was not available at the moment of the study. The results indicate guidelines for the formulation of academic policy for decision making and execution of actions designed to correct and better the functioning of university dependencies. These indicators, and the models from which they derive could be applied to other academic units in the university or to other higher educational institutions that offer academic degrees or titles. The conclusion is that the measurement and evaluation of management indicators are effective practices in implementing auto-regulation and control in institutions of higher education.

Key words: Indicators, efficiency, academic units, universities.

\section{Introducción}

Gerencia de Sistemas Conductuales es un modelo que ha mostrado su aplicación en el área de cambio organizacional (Yáber, Malott y Valarino, 1998: 279) y que puede emplearse en combinación con un enfoque sistémico (Contraloría General de la República de Venezuela, 1999: 40) , para mejorar las funciones gerenciales, en particular la gestión de control. En tal sentido, el objetivo del presente trabajo consiste en la aplicación del modelo de Gerencia de Sistemas Conductuales y un enfoque sistémico de organización y gestión, en el diseño, desarrollo, aplicación y evaluación de indicadores de desempeño clave en una unidad académica universitaria venezolana, concretamente en la Universidad Simón Bolívar, una universidad pública venezo- lana, donde se seleccionó intencionalmente, el decanato de estudios de postgrado. Este decanato cumplió 30 años de fundado el año 2001. Para ese año ofrecía a través de sus 27 coordinaciones, 61 programas de postgrado en las áreas de ciencias básicas, ingeniería, tecnología, ciencias sociales y humanidades.

Se empleó la metodología de la Gerencia de Sistemas Conductuales que incluye las fases de: análisis, especificación, diseño, implementación, evaluación y reciclaje. Durante el análisis se ubicó el organigrama de la institución para describir y relacionar los elementos de su estructura organizacional y la del decanato de estudios de postgrado. Luego se desarrolló el mapa orgánico de procesos empleando el análisis de pasos, actividades y tareas (Rummler y Brache, 1995: 16). Seguidamente se identificaron los 
procesos de dirección, claves y de apoyo del decanato. En la etapa de especificación se empleó el sistema global de desempeño (Malott, 1999: 34). Se identificaron y analizaron los componentes del sistema conductual (decanato de estudios de postgrado). Estos insumos facilitaron el desarrollo del esquema de análisis sistémico de organización y gestión propuesto por la Contraloría General de la República de Venezuela, para el desarrollo del sistema de control interno de gestión. En la etapa de diseño se seleccionó la intervención de acuerdo a lo obtenido en las fases de análisis y especificación. Posteriormente en la fase de implementación se desarrollaron los indicadores de gestión y se recolectaron los resultados obtenidos. En la fase de evaluaciónse analizaron los resultados, que sirvieron de insumos para esbozar las mejoras que se estiman implantar en la fase de reciclaje.

\section{El Enfoque de Sistemas Conductuales}

Gerencia de Sistemas Conductuales (G.S.C.), es un enfoque para el cambio en las organizaciones, con el cual se diseñan, implantan, evalúan y reciclan sistemas en los que participan seres humanos, con el propósito de asegurar que las organizaciones logren sus metas (Yáber, Malott y Valarino, 1998: 279). Esta perspectiva incluye estrategias para ayudar a las organizaciones a desarrollar programas de intervención relacionados con el cambio y el Desarrollo Organizacional (D.O.) (Malott, 1999: 33). En la G.S.C. se concibe el cambio principal- mente, como un proceso de innovación y adopción de nuevas ideas y comportamientos donde se persigue reducir las diferencias entre el desempeño actual y el deseado, mediante intervenciones planeadas dirigidas al individuo, equipos, procesos 0 al sistema organizacional, para alcanzar los objetivos que se han trazado conjuntamente con el cliente.

El proceso de la G.S.C. comprende las fases de: análisis, especificación, diseño, implementación, evaluación y reciclaje. Durante el análisisse identifican los elementos que constituyen el sistema conductual (individuo, departamento, proceso, organización) y las relaciones que existen entre ellos. La segunda fase, especificación, consiste en determinar los objetivos de desempeño que se desean para el sistema conductual. Posteriormente, se diseña la intervención, especificando el tipo y el plan de seguimiento. Luego se implementa el programa de cambio en la organización, de acuerdo a lo especificado en el diseño, para posteriormente evaluarlo. La evaluación consiste en comparar los resultados obtenidos con la intervención, en relación a lo que se había determinado como objetivo deseable. Finalmente, y de acuerdo a la evaluación, se procede a reciclar (reiniciar el proceso desde la fase de análisis) hasta que se alcancen los objetivos previamente definidos en el plan de cambio en la organización (Yáber, Malott y Valarino, 1998: 281).

La G.S.C. puede aplicarse para mejorar las funciones administrativas que realizan los directores, gerentes y supervisores en la organización. Estas funciones son: planificación, organización, di- 
rección y control. Esta clasificación es una pequeña variación de lo propuesto en 1916 por Henri Fayol, quién señaló que todos los gerentes desempeñaban cinco funciones administrativas: planear, organizar, ordenar, coordinar y controlar (Fayol, 1998: 132). Contemporáneamente, las funciones de ordenar y coordinar se han agrupado bajo las funciones de "dirección" o de "control".

Planificación es la función que cumplen los gerentes para establecer las metas que deben alcanzar las organizaciones o sus unidades y los cursos de acción que se estiman pertinentes y necesarios para lograr estas metas. Organización es la función que se ejecuta con el propósito de ordenar y distribuir el trabajo, la autoridad y los recursos necesarios para alcanzar las metas que se ha propuesto una unidad o sistema organizacional. Dirección es la función dentro de la gestión, que implica mandar, influir y motivar a los colaboradores para que realicen las tareas que llevarán a lograr las metas de la unidad y de la organización. Finalmente, la función de control, comprende el monitoreo de las actividades incluidas en los planes estratégicos, operativos y tácticos de la organización, con el fin de examinar si existen discrepancias entre lo que se cumple y lo que se debería cumplir para alcanzar las metas y objetivos de la organización y sus unidades, con el propósito de instrumentar los mecanismos necesarios para corregir el rumbo o mantenerse en lo pautado de acuerdo al plan previamente trazado. El control puede ser interno o externo a la unidad u organización. Igualmente, el control puede ser previo, concurrente o posterior a la gestión. Los administrado- res, gerentes o gestores, cumplen parcial o totalmente con cada una de estas funciones, cuando se encuentran en la dirección general de una organización o cuando cumplen con estas responsabilidades en alguna de sus unidades, departamentos o gerencias.

\section{Fundamentación Legal del Control Externo Universitario}

Las universidades como organizaciones requieren de una dirección que cumpla cabalmente con las funciones que se acaban de delinear, para alcanzar con excelencia las metas y objetivos que se proponen. La gestión de las universidades, como la de cualquier otra organización que se dedique a prestar servicios o producir bienes, requiere de sus autoridades y dirección media, el ejercicio de las funciones de planificación, organización, dirección y control. El marco regulador de cada país coloca las condiciones límite para la gestión de las autoridades universitarias.

En las universidades públicas nacionales de Venezuela, la función de control externo la ha ejercido fundamentalmente la Contraloría General de la República. El control ha estado básicamente relacionado con la auditoria de la ejecución anual del presupuesto asignado a las universidades. La Contraloría General de la República, a través de la contraloría interna de cada universidad, ha llevado a cabo el control previo de las órdenes de pago de las unidades y presenta a final de cada año, un conjunto de propuestas orientadas a sugerir mejoras, e incluso proponer sanciones, para garantizar el cumplimiento de 
la ejecución presupuestaria de acuerdo a las normas establecidas internamente $u$ originadas de organismos externos a la universidad, como el Consejo Nacional de Universidades.

Aunque algunas universidades cuentan con consejos superiores 0 instancias equivalentes que deberían vigilar y ofrecer información de retorno a la institución sobre el cumplimiento de sus planes y programas, y a pesar que las universidades deben presentar informes anuales a consideración de instancias superiores, estos mecanismos de control externo no han sido realmente efectivos para determinar si las universidades se encuentran cumpliendo a cabalidad con su misión. Aún en el caso que estos mecanismos funcionen, resulta problemático emitir un juicio valorativo de la institución, pues no se cuenta con indicadores cuali-cuantitativos de la gestión, que permitan evaluar año tras año, si el desempeño de la institución mejora, o al menos se mantiene dentro de lo contemplado en sus planes de desarrollo.

El gobierno de Venezuela aprobó en 1995 (República de Venezuela, 1995) la ley para la Contraloría General de la República que proporcionaba un marco legal para el ámbito de su competencia. Esta ley, sin renunciar a su labor fiscalizadora, añadía la función de consultoría al quehacer de la Contraloría. Entre sus atribuciones estaba apoyar a las instituciones para que éstas realizaran auditorías internas de gestión, donde cada organismo autoevaluara el cumplimiento de su misión y colaborara con la contraloría en su actividad de control.

Las organizaciones públicas, y en particular las universidades, requerían dos acciones para cumplir con la ley. En primer lugar, desarrollar unidades internas de control de gestión dependientes de la contraloría interna de cada institución y en segundo término, cada unidad académica y administrativa en cada universidad tendría que formular su misión, visión y plan estratégico, dentro del marco general de visión, misión y plan estratégico de la universidad y además, desarrollar los indicadores de su gestión (Contraloría General de la República de Venezuela, 1999: 40).

En la nueva Ley orgánica de la Contraloría General de la República y del Sistema Nacional de control fiscal (República Bolivariana de Venezuela, 2001: 321 ), en su artículo 35, se define el control interno como un "... sistema que comprende el plan de organización, las políticas, normas, así como los métodos y procedimientos adoptados dentro de un ente u organismo sujeto a esta Ley, para salvaguardar sus recursos, verificar la exactitud y veracidad de su información financiera y administrativa, promover la eficiencia, economía y calidad en sus operaciones, estimular la observancia de las políticas prescritas y lograr el cumplimiento de su misión objetivos y metas".

Esta ley igualmente destaca en su artículo 37, que cada entidad elaborará los indicadores de gestión y otros instrumentos o métodos para el funcionamiento del sistema de control interno.

Desde hace algunos años se viene trabajando en propuestas de indicadores de gestión (Soriano, 1997:1). Esta iniciativa impulsó un interés colectivo en las universidades de Venezuela que motivó el mayor interés del Estado en este aspecto, quién lo ha canalizado 
con la aprobación de un proyecto para el desarrollo de indicadores de gestión para las universidades, patrocinado por el Consejo Nacional de Investigaciones Científicas y Tecnológicas (ahora FONA$\mathrm{CIT}$ ) y en el cual trabajan conjuntamente tres universidades nacionales incluyendo a la Universidad Simón Bolívar (Salcedo, 1999: 63).

El requerimiento del Estado venezolano de asegurar el cumplimiento de la Constitución de la República Bolivariana de Venezuela (2000) en lo referente a su artículo 103 sobre la calidad de la educación (Villarroel, 2000: 8), unido al interés de las universidades públicas venezolanas de mostrar el cumplimiento de sus objetivos y metas, incluyendo el proyecto de indicadores nacionales de gestión universitaria, proporcionan argumentos para justificar este proyecto sobre indicadores de desempeño clave para las distintas unidades académicas en universidades nacionales.

\section{La Aplicación del Modelo en la Universidad Simón Bolívar}

El Diagrama 1 expresa el sistema general de la unidad académica en la cual se está aplicando el modelo CGS. En el mismo puede apreciarse que la misión del decanato de estudios de postgrado es la formación y acreditación de profesionales con grados académicos de cuarto nivel, siendo los resultados o productos los egresados de los programas académicos de postgrado: técnicos superiores especialistas, especialistas profesionales, magíster y doctores. Sus clientes son: el estado venezolano y organizaciones públicas o privadas que se dedican a producir bienes o prestar servicios. También los profesionales por iniciativa individual son clientes de este sistema conductual. En el caso de la Universidad Simón Bolívar y su decanato de postgrado, el estado es el más importante cliente y proveedor de los recursos que son necesarios para el proceso. Estos recursos son el personal académico y de apoyo, los recursos financieros, los recursos tecnológicos, el ambiente físico, la infraestructura y la dotación de equipos y suministros. El proceso, destaca los aspectos que se requieren para la formación del recurso humano de cuarto nivel.

Los procesos claves corresponden a programas académicos, así como los procesos de dirección (planificación, captación y asignación de recursos y evaluación del desempeño) y los diez procesos de apoyo, son los siguientes: Ingresos, prórrogas, reingresos, tesis, inscripciones, financiamientos, equivalencias, procesos extemporáneos, apertura de programas y acreditación de programas. El proceso proporciona información de retorno a los insumos o recursos con datos acerca de cuánto tiempo se toma en formar a un egresado, así como el costo y la calidad de los procesos, con el propósito de ayudar al sistema conductual a tomar decisiones y acciones para corregir o seguir el rumbo que se ha propuesto. Los clientes indican su satisfacción con los servicios que prestan los egresados y ponderan su calidad a través de información de retorno del cliente. El sistema estaría incompleto sin la consideración de la competencia, que son las universidades $y$ otros institutos $u$ organizaciones que prestan servicios de educación de postgrado y que compiten con el decanato de 


\section{Diagrama 1 \\ Sistema Global de Desempeño del decanato de Estudios de Postgrado}

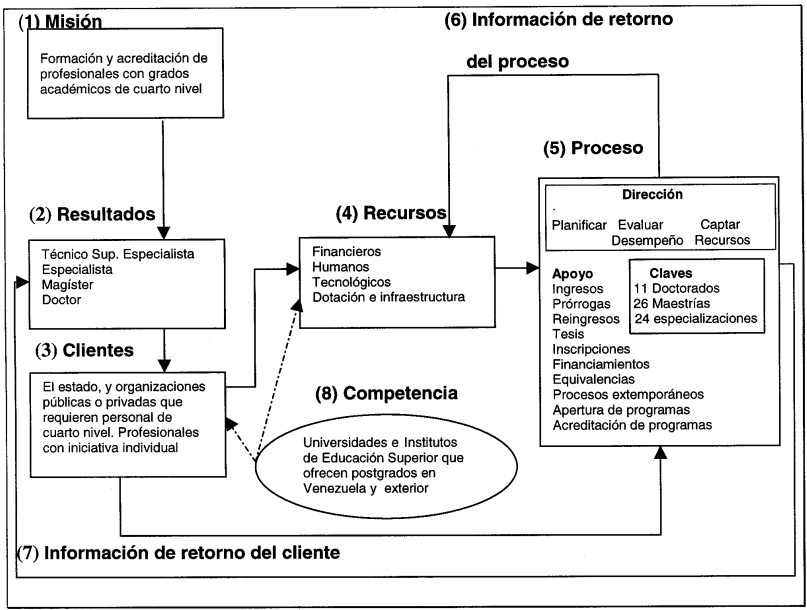

Fuente: Elaboración Propia.

estudios de postgrado por los clientes y recursos que se requieren para llevar a cabo satisfactoriamente el proceso de formación.

El análisis funcional realizado en esta etapa de especificación permitió constatar que la unidad presentaba debilidades en sus procesos de dirección pues no contaba con recursos para evaluar su desempeño como una unidad. Por ello se decidió en la etapa de diseño, desarrollar un sistema de indicadores que facilitase el desarrollo de un sistema de control interno de gestión. Para desarrollar este sistema se procedió entonces en la etapa de implementación, a aplicar el esquema de organización y gestión (Diagrama 2). Las categorías que definen el sistema conductual del decanato de estudios de postgrado y que permiten evaluar su desempeño son: efecto, impacto, eficacia, eficiencia, economía y calidad.
Las variables requeridas para calcular los indicadores de gestión para la unidad académica son: a) año fiscal; b) nuevos ingresos a programas de doctorado; c) nuevos ingresos a programas de maestría; d) nuevos ingresos a programas de especialización; e) matrícula anual promedio de postgrado; f) egresados de especialización; g) egresados de maestría; h) egresados de doctorado; i) programas de postgrado vigentes; j) programas de postgrado acreditados $y$ k) programas de postgrado cofinanciados.

Las mediciones y evaluación de los indicadores para estas categorías se presentan a continuación.

Efecto: se refiere a la repercusión inmediata en el entorno de los resultados, productos o servicios prestados por una organización o unidad. Para el decanato de estudios de postgrado, una unidad 


\section{Diagrama 2 \\ Enfoque sistémico de organización y gestión del Decanato de Estudios de Postgrado}

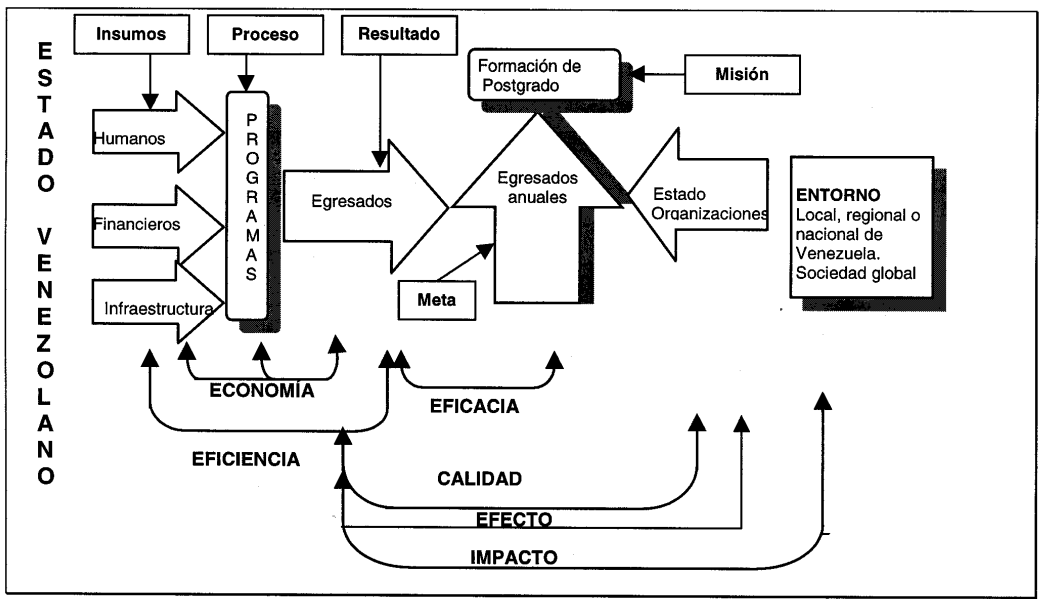

Fuente: Elaboración Propia.

académica de la Universidad Simón Bolívar, el efecto se refiere al número de egresados por año fiscal. En el Gráfico 1, se puede examinar la evolución del indicador de efecto desde 1996 hasta el año 2000.

De la inspección visual del Gráfico 1 se puede concluir que los egresados vienen aumentando a través del tiempo considerado, con la excepción del año de 1998 donde se presenta el número de egresados más bajo en la serie de 5 años considerada.

Impacto: explicita la repercusión a mediano y largo plazo en el entorno de los resultados, productos o servicios prestados por la organización o unidad. Para el decanato de estudios de postgrado el impacto se refiere al número de egresados por bloques de cuatro años fiscales desde 1973 hasta el año 2000. En el Gráfi- co 2 se puede examinar la evolución en el tiempo del indicador de impacto.

La serie muestra claramente un crecimiento cuantitativo sostenido en el número de los egresados de postgrado desde 1973 hasta el año 2002.

Los resultados sugieren que el decanato de estudios de postgrado ha tenido un efecto y un impacto en la comunidad al otorgar títulos de postgrado sostenidamente desde 1973 hasta el año 2000. En 1998 hubo una reducción en el número de graduados que podría atribuirse a retrasos en el tiempo de graduación, reducción de nuevos ingresos en los años anteriores o disminución de la matrícula por razones económicas. Estos puntos requieren de nuevos estudios.

Eficacia: es la relación que existe entre la cantidad de productos, servicios o resultados que se producen y las metas 


\section{Gráfico 1 \\ Efecto. Número de egresados por año fiscal}

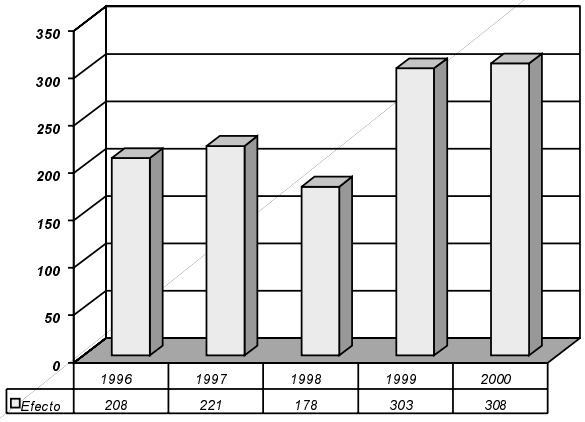

Fuente: Elaboración Propia.

Gráfico 2

Impacto. Número de egresados acumulados por cuatro años fiscales

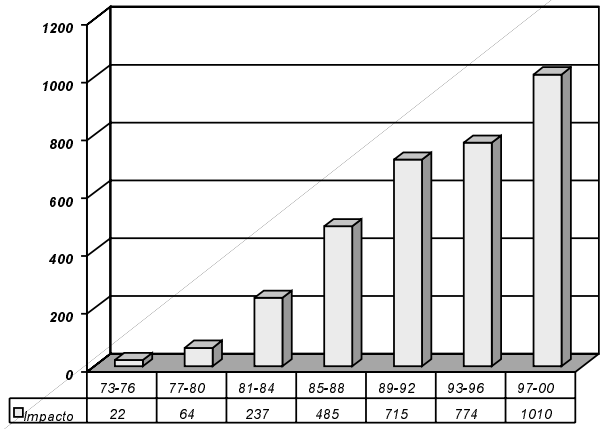

Fuente: Elaboración Propia.

programadas. En el decanato, la eficacia se midió por el número de egresados por año fiscal dividido entre la meta programada de egresados para ese año fiscal. Hasta la fecha no se habían establecido metas de graduados por año, por lo que no se pudo medir este parámetro de gestión. A partir del año 2001 se estimó una cifra de egresados para el año fiscal a ser comparada con el número de egresados obtenidos a final de año. La cifra de egresados se estimó empleando como variable predictora, los egresados en los cinco años fiscales previos y la variable de criterio, el número de egresados estimado para el año 2001.

Eficiencia: es la relación entre los resultados y la cantidad de recursos 
empleados para su obtención. En el decanato de estudios de postgrado se midió la eficiencia, dividiendo número de graduados por año entre el número de admitidos tres años antes (especialización y maestría) o el número de admitidos cuatro años antes (para el caso del doctorado), multiplicado por 100. Estas cifras de 3 y 4 años corresponden al tiempo estimado en la normativa nacional de postgrado para la culminación de los programas de especialización, maestría y doctorado. La eficiencia de los programas de postgrado se puede examinar en los Gráficos 3 (especialización), 4 (maestría) y 5 (doctorado).

La eficiencia de los programas de especialización muestra un comportamiento irregular, donde aumenta y se reduce la eficiencia anualmente, con oscilaciones que van del 30 al $94 \%$.

La eficiencia de los programas de maestría creció leve pero sostenidamente entre 1996 y 1999. En el año 2000, sin embargo, se aprecia una disminución significativa de este indicador.

La eficiencia de los programas de doctorado se incrementó sostenidamente desde 1996 hasta el año 2000, donde se destaca un incremento notable de 1996 a 1997.

La eficiencia, el segundo indicador, muestra diferencias de acuerdo a los programas. La eficiencia del doctorado mostró variaciones en los dos primeros años y luego existe una tendencia creciente sostenida que apunta en la dirección deseada de graduar doctores en el tiempo estipulado de cuatro años. La eficiencia de la especialización muestra variaciones pero en conjunto refleja una tendencia en su crecimiento llegando hasta 94\% en 1999. La eficiencia de la maestría muestra un comportamiento relativamente estable de $43 \%$ con ligeras variaciones. Al examinar los tres indicadores se constata que es preciso prestar mayor atención a la maestría, ya que constituye el programa de postgrado que presenta menor eficiencia.

Calidad: se refiere a la cantidad, grado y oportunidad en que el servicio o producto satisface la necesidad del beneficiario o usuario. En el decanato se calculó la calidad, dividiendo el número total de programas acreditados por el Consejo Nacional de Universidades (organismo nacional que certifica que los programas de postgrado cumplen con los requisitos mínimos para operar nacionalmente), vigentes para el año fiscal, entre el total de programas, multiplicado por 100 . El indicador de calidad puede revisarse en el Gráfico 6.

La calidad medida como porcentaje de programas acreditados es todavía baja y se mantiene oscilando entre el $27 \%$ y el $36 \%$ de los programas vigentes para la serie de tiempo considerada.

La calidad de los programas muestra un comportamiento homogéneo a lo largo de los cinco años y se ubica en un promedio de $33 \%$ con un decrecimiento en el año 2000 que ocurre como resultado del vencimiento de la acreditación de algunos programas y el aumento de programas ofrecidos en el año 2000. Es necesario que el decanato desarrolle una estrategia para aumentar rápidamente el porcentaje de programas acreditados, requisito indispensable para mejorar la calidad de su gestión. La calidad se definió 


\section{Gráfico 3}

Eficiencia en la especialización (egresados año fiscal / \# nuevos inscritos 3 años fiscales previos) $\times 100$

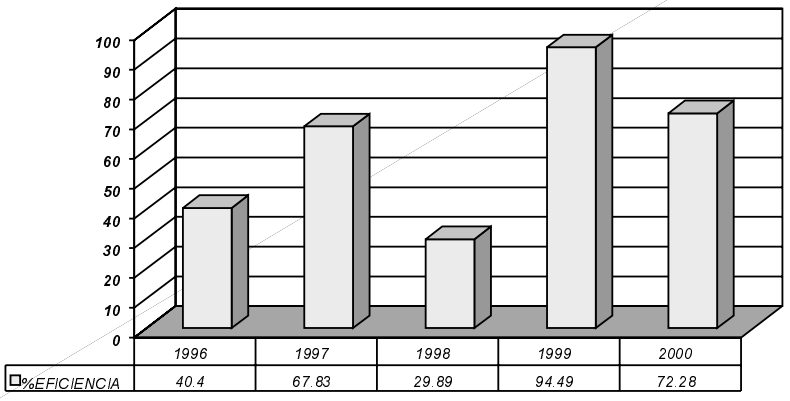

Fuente: Elaboración Propia.

\section{Gráfico 4}

Eficiencia en la maestría. (egresados año fiscal / \# nuevos inscritos 3 años fiscales previos) $\times 100$

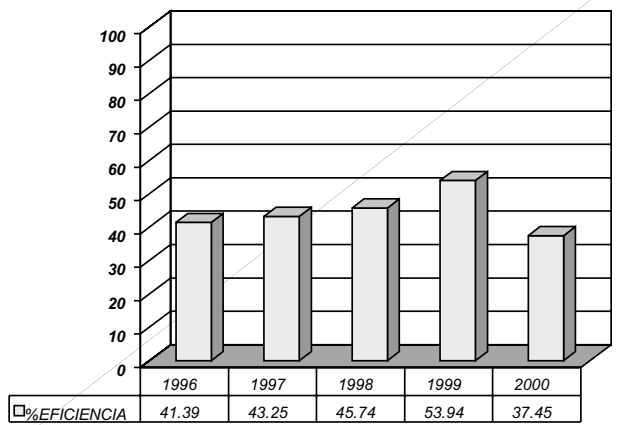

Fuente: Elaboración Propia.

como el porcentaje de programas acreditados por una agencia del gobierno venezolano. Este es un indicador cuantitativo que tiene valor comparativo con el resto de las instituciones venezolanas que ofrecen programas de postgrado, pero no agota las posibilidades de evaluación de la calidad como categoría de gestión. Es posible y deseable generar otros indicadores de calidad tanto cuantitativos como cualitativos (calidad del egresado, calidad del programa y calidad de los insumos) para enriquecer y complementar los formulados en este trabajo. 


\section{Gráfico 5 \\ Eficiencia en el doctorado. (egresados año fiscal / \# nuevos inscritos 4 años fiscales previos) $\times 100$}

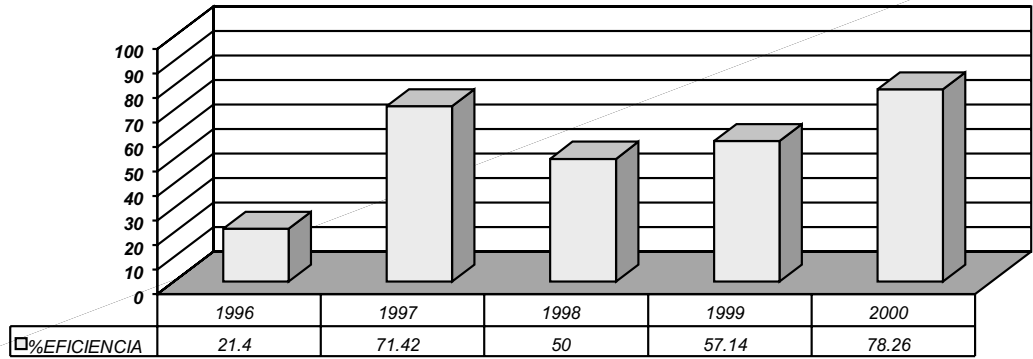

Fuente: Elaboración Propia.

Gráfico 6

Calidad. Porcentaje anual de programas de postgrado acreditados

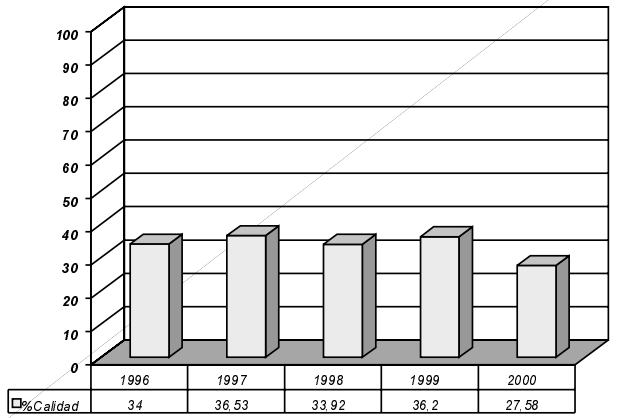

Fuente: Elaboración Propia.

Economía: consiste en utilizar apropiadamente los insumos (calidad y cantidad), con el menor costo posible, con relación a los programas de la organización y con las condiciones que presenta el mercado. En el decanato de estudios de postgrado se calculó la economía, dividiendo el número total de programas amparados por convenios de co-financiamiento vigentes para el año fiscal, divididos entre el número total de programas vigentes para el año fiscal, multiplicados por 100 (Ver Gráfico 7).

El número de programas cofinanciados es relativamente baja a través del tiempo, aunque se observaron mejoras 
Gráfico 7

Economía. Porcentaje anual de programas de postgrado co-financiados

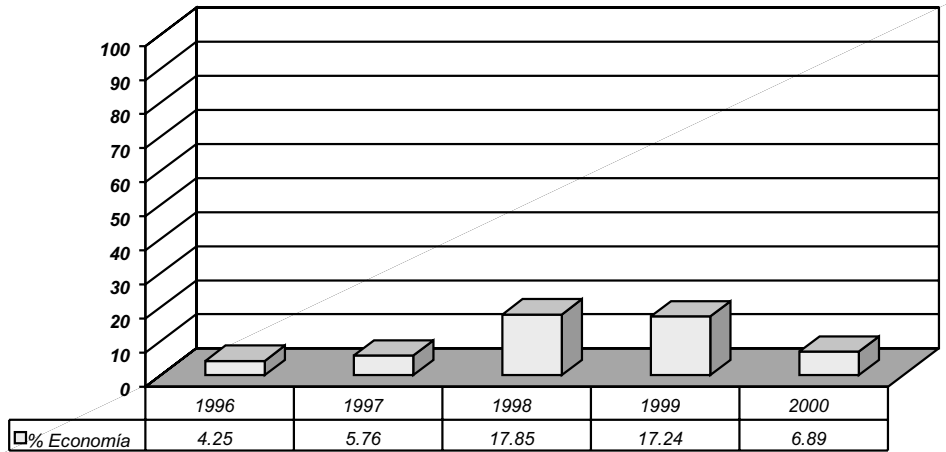

Fuente: Elaboración Propia.

en los años 1998 y 1999 donde el 17\% de los programas de postgrado estuvieron cofinanciados.

La economía de la gestión del postgrado, entendida como el porcentaje anual de programas co-financiados, es quizás la mayor debilidad de la gestión de la unidad, pues el porcentaje de programas acreditados es relativamente bajo. Durante dos años (98 y 99) estos porcentajes aumentaron de $5 \%$ a $17 \%$, pero luego decayeron de nuevo. Esto se debe a que las principales agencias de financiamiento públicas (CONICIT y FUNDAYA$\mathrm{CUCHO}$ ) redujeron, eliminaron o terminaron sus financiamientos a algunos programas en estos cinco años. En un plan de gestión que contemple oportunidades de mejora, el mejoramiento del indicador de economía tendría que ocupar un lugar fundamental en los esfuerzos de gestión de la unidad y de la organización.

\section{Conclusiones}

A partir de los resultados obtenidos con el cálculo de los indicadores, la inspección visual de los gráficos y el análisis de los resultados, se pueden formular las siguientes consideraciones y conclusiones.

Los indicadores presentados ofrecen un panorama amplio del desempeño del decanato de estudios de postgrado como unidad académica de la universidad. De los seis indicadores propuestos, el indicador de eficacia no se pudo calcular. La dependencia académica ya ha tomado medidas para que ésta se pueda calcular en el futuro una vez que se planifique cuantas personas planifica graduar cada año. Tres de los cinco indicadores calculados muestran incrementos o mejoras a lo largo del lapso de tiempo considerado. El desempeño de la unidad mejora con el tiempo en términos de efecto, 
impacto y eficiencia. Dos indicadores, el de calidad y el de economía, indican la necesidad de tomar medidas inmediatas para corregir tendencias no deseadas. Específicamente, el decremento en el indicador de calidad se debe en parte, al vencimiento en el lapso de acreditación de varios programas y a un crecimiento muy lento en la cantidad de programas acreditados. Esta tendencia puede revertirse iniciando un plan conjunto entre el decanato y las coordinaciones académicas, orientado a completar los pasos que se requieren para solicitar la re-acreditación de los programas que tienen lapsos vencidos, así como acreditar los programas vigentes que así lo necesiten. La tendencia a desmejorar en el indicador de economía, se debe parcialmente a la suspensión temporal de financiamiento a la actividad de postgrado de la universidad por parte de agencias del gobierno durante los años 1999 y 2000. Esta tendencia tiende a revertirse en razón que el gobierno ha recomenzado su plan de financiamiento en el año 2001 para estudiantes y programas de postgrado. Se requiere, sin embargo, de más iniciativas por parte de las coordinaciones académicas de los programas para solicitar financiamiento externo en entidades nacionales e internacionales.

Los indicadores calculados y analizados en el presente trabajo, tienen varias ventajas como son su sencillez, cobertura, generación de nuevos indicadores y valor comparativo. Su sencillez se deriva del hecho que requieren variables de fácil obtención (nuevos ingresos, egresados, y programas vigentes por año fiscal entre otros) y el cálculo de los indicadores se puede realizar manualmente.
Una segunda ventaja es su cobertura, pues se consideran la mayoría de los indicadores de gestión (una posible excepción es la categoría de pertinencia). La generación de otros indicadores, combinando los calculados en el presente trabajo, es otra ventaja adicional. Por ejemplo, se pueden sumar los porcentajes promedios de eficacia y eficiencia para obtener un indicador de productividad. Se pueden promediar los porcentajes de eficiencia de especialización, maestría y doctorado para obtener un indicador de eficiencia global. Por otra parte, se puede promediar la suma de los porcentajes de eficacia, eficiencia, calidad y economía, para generar un indicador global de desempeño. Finalmente, los indicadores tienen valor comparativo. Pueden emplearse para comparar unidades de postgrado de distintas universidades con propósitos de "benchmarking". Por ejemplo, se puede emplear el indicador de eficiencia global arriba señalado, para comparar unidades de postgrado de diversas universidades.

Los enfoques empleados se aplicaron en una unidad académica de una organización que presta servicios educativos, pero podrían aplicarse a uno de sus programas o proyectos, a toda la organización, a otras organizaciones que prestan servicios e incluso a organizaciones que producen bienes en lugar de prestar servicios, lo que sugiere su amplio espectro de aplicación.

Los indicadores del decanato como unidad y sus criterios son insuficientes. Se sugiere indagar sobre indicadores $y$ criterios de desempeño de otras instituciones, que permitan comparar lo obtenido en la Universidad Simón Bolívar y que 
contribuyan a cambiar y mejorar los indicadores existentes. Se sugiere en futuros estudios enriquecer los indicadores cuantitativos, agregando indicadores cualitativos para cada una de las categorías de gestión establecidas en este estudio. Finalmente, es necesario formular los indicadores para cada programa de postgrado con el fin de establecer la línea de base sobre la cual las coordinaciones académicas trabajarán para mejorar su desempeño. La combinación de enfoques (Yáber, Malott y Valarino, 1998: 279; Contraloría General de la República de Venezuela, 1999: 40) y sus resultados, pueden servir de ejemplo para otras unidades académicas que se encuentren en el proceso de desarrollar sistemas de control interno de la dirección en las diversas dependencias de los institutos de educación superior. Medir y evaluar indicadores de gestión constituyen prácticas para instrumentar la autorregulación y el control interno de la gestión en las instituciones de educación superior.

\section{Bibliografía Citada}

Contraloría General de la República de Venezuela (1999). Metodología para la auditoría de gestión. Cuadernos de Auditoria No. 3. Caracas: Ediciones de la Contraloría General de la República.

Fayol, Henri (1998). Administración industrial y general. México: Herrero Hermanos, S.A.

Malott, María (1999). Creating lasting organizational changes. Performance improvement. Año $38, \mathrm{~N}^{\circ} 1$, USA pp. 33-36.
República de Venezuela (1995): Ley Orgánica de la Contraloría General de la República. Gaceta Oficial N 5.017, Extraordinario. diciembre 13. Venezuela.

República Bolivariana de Venezuela (2001). Ley Orgánica de la Contraloría General de la República y del Sistema Nacional de Control Fiscal. Gaceta Oficial N 37.347. diciembre 17. Venezuela.

República Bolivariana de Venezuela (2000). Constitución de la República Bolivariana de Venezuela, 1999. Gaceta oficial N 5.453, Extraordinario. marzo 24. Venezuela.

Rummler, G., y Brache, A. (1995). Improving performance: How to manage the white space on the organization chart. San Francisco, USA: JosseyBass Inc. Publishers.

Salcedo, Hernando. (1999). Indicadores de gestión para las universidades venezolanas: un proyecto de alcance nacional. Agenda Académica. Año 6, $\mathrm{N}^{\circ} 1$. Venezuela pp. 63-91.

Soriano, Myriam. (coordinadora) (1997). Propuesta sobre indicadores de gestión. Informe técnico. Universidad Simón Bolívar: comisión de planificación. Venezuela.

Villarroel, César (2000). Calidad Universitaria e indicadores de gestión. Gaudeamus, Año 1, $\mathrm{N}^{\circ} 3$, Venezuela, pp. 8-10.

Yáber, Guillermo, Malott, María y Valarino, Elizabeth (1998). Gerencia de sistemas conductuales y cambio organizacional. Revista Latinoamericana de Psicología, Año $30 \mathrm{~N}^{\circ}$ 2, Colombia, pp. 279-291. 